\title{
Obstacle Avoidance for an Extremum Seeking System using a Navigation Function
}

\author{
Hans-Bernd Dürr, Miloš S. Stanković, Dimos V. Dimarogonas, Christian Ebenbauer, Karl Henrik Johansson
}

\begin{abstract}
The problem of autonomously steering a vehicle to a destination point, while avoiding obstacles, is considered. The vehicle is modeled as a single-integrator in the plane and it is assumed that the obstacles are unknown a priori. The control law is an extremum seeking algorithm, which steers the vehicle to the minimum of a navigation function. In this framework, obstacle avoidance and practical uniform convergence to a destination point for almost all initial conditions is proven. The theoretic results are illustrated using numerical examples.
\end{abstract}

\section{INTRODUCTION}

In many applications dealing with autonomous robots, one important task is to steer the robot to a certain destination while avoiding obstacles. In this paper, we deal with such a problem from a theoretic perspective. We consider a singleintegrator vehicle in two dimensions. The goal is to construct a feedback which steers the vehicle to a destination point while avoiding obstacles. We additionally assume that the vehicle can only measure the Euclidian distance to the obstacles and to the destination point.

In order to solve this problem, we propose a method based on a navigation function approach which was introduced in [5]. The approach is based on a function with particular properties. We denote it as navigation function $\varphi$ and its construction is based on a combination of the Euclidian distances of the vehicle to the obstacles and to the destination point. The domain of $\varphi$ will be denoted as the free space and is a compact subset in $\mathbb{R}^{2}$. One can think of the free space as the set where the vehicle is allowed to move. In [5] it is shown that the trajectories of the gradient-system $\dot{x}=-\nabla \varphi(x)$ converge to a given destination point for almost all initial conditions and avoid obstacles at the same time (see Proposition 2.4 in [5]). The construction procedure for $\varphi$ yields four properties which are very important for the theoretical analysis of the proposed procedure. First, $\varphi$ is analytic. Second, it is polar which means that it admits a

Hans-Bernd Dürr and Christian Ebenbauer are with the Institute for Systems Theory and Automatic Control, University of Stuttgart, Germany and are supported by the Deutsche Forschungsgemeinschaft (Emmy-Noether-Grant, Novel Ways in Control and Computation, EB 425/2-1, and Cluster of Excellence in Simulation Technology, EXC 310/1). Miloš S. Stanković is with the Innovation Center, School of Electrical Engineering, University of Belgrade, Serbia and is supported by the EU Marie Curie CIG and Serbian Ministry of Science and Technology. Dimos V. Dimarogonas and Karl Henrik Johansson are with the ACCESS Linnaeus Center, School of Electrical Engineering, Royal Institute of Technology, 100-44 Stockholm, Sweden; Dimos V. Dimarogonas is also affiliated with the KTH Center for Autonomous Systems. They are supported by the Knut and Alice Wallenberg Foundation, Swedish Research Council and the EU HYCON2 NoE; E-mail: hans-bernd.duerreist.uni-stuttgart.de, milstalkth.se, dimosekth.se, celist.uni-stuttgart.de, kallejekth.se. unique minimum in the free space. It turns out that this is the destination point for the vehicle. Third, it is a Morse function (see [4], [10]) which implies that all critical points of $\varphi$ are non-degenerate. Fourth, the function $\varphi$ admits a constant value at the boundary of the workspace. This property is called admissible. The authors in [5] also point out that there is no smooth gradient vector field which yields globally convergent trajectories to the destination point in the presence of obstacles but there will always exist additional steady states. Fortunately, since these points are saddle points, almost convergence can be guaranteed, i.e. convergence to the destination points for almost all initial conditions.

There are many approaches in the literature (see e.g. [7]) which are based on a navigation function. For an extension to the multi-agent case see e.g. [2] and [8]. However, in these references, it is assumed that the gradient of $\varphi$ is available. It turns out that for a moving vehicle the gradient of a distance is difficult to measure physically although there are sensors which measure the distance accurately. In order to solve this problem, we apply extremum seeking (see e.g. [3], [6], [14]). Extremum seeking is a powerful method to steer a dynamical system to the minimum of a nonlinear map by only measuring its values. In other words, it is a method to estimate online the gradient and feed it back into a dynamical system which then moves into the direction of the steepest descent. In an earlier work [3] a novel analysis method for extremum seeking vehicles was introduced. It is based on a Lie bracket trajectory approximation. With this method it is possible to state qualitatively how the trajectories of the extremum seeking system evolve in the large.

The main idea of this paper is to combine the idea of Lie bracket trajectory approximation for extremum seeking systems introduced in [3] with the navigation function approach in [5]. An extensive simulation study with a similar approach using potential functions was done in [16].

The main contribution is twofold. First, we calculate an approximating system of a single-integrator extremum seeking system. We prove almost convergence to the destination point in the free space and show that obstacle avoidance is guaranteed. Second, we show how the properties of the approximated system translates to the extremum seeking system. It turns out that obstacle avoidance and almost practical convergence can be guaranteed for the extremum seeking system in the free space. To the authors best knowledge, no methodology with similar strong convergence results is known.

The remainder of this paper is structured as follows. In 
Section II, we introduce the mathematical preliminaries and the problem setup. In Section III, we present the main results where we state almost convergence, invariance of the workspace for the Lie bracket system and based on this, we prove almost practical convergence and invariance of the extremum seeking system. In Section IV, we show a numerical example and summarize the results in Section V.

\section{Preliminaries}

\section{A. Notation}

The following notation will be used throughout the paper. The intervals of real numbers are denoted by $(a, b)=\{x \in$ $\mathbb{R}: a<x<b\},[a, b)=\{x \in \mathbb{R}: a \leq x<b\}$ and $[a, b]=$ $\{x \in \mathbb{R}: a \leq x \leq b\}$. The norm $|\cdot|$ denotes the Euclidian norm. The determinant of a matrix $A \in \mathbb{R}^{n \times n}$ is denoted as $\operatorname{det}(A)$ and its trace is denoted by $\operatorname{trace}(A)$. A matrix $A \in \mathbb{R}^{n \times n}$ is said to be positive semidefinite (definite), if $x^{\top} A x \geq 0\left(x^{\top} A x>0\right)$ for all $x \in \mathbb{R}^{n} \backslash\{0\}$. A matrix $H \in$ $\mathbb{R}^{n \times n}$ is Hurwitz if its eigenvalues are in the complex open left half-plane. The interior of a compact set $S$ is denoted as $\operatorname{int}(S)$ and its boundary as $\partial S$. We define the open ball $B\left(x_{0}, \epsilon\right)$ as the set $B\left(x_{0}, \epsilon\right)=\left\{x \in \mathbb{R}^{n}:\left|x-x_{0}\right|<\epsilon\right\}$. Let $f: \mathbb{R}^{n} \rightarrow \mathbb{R}$ be twice continuously differentiable. Then

$$
\begin{aligned}
& \nabla_{i} f(x)=\frac{\partial f(x)}{\partial x_{i}}, \nabla_{i, j} f(x)=\frac{\partial^{2} f(x)}{\partial x_{i} \partial x_{j}}, \nabla_{i}^{2} f(x)=\frac{\partial^{2} f(x)}{\partial x_{i}^{2}}, \\
& \nabla f(x)=\left(\frac{\partial f(x)}{\partial x}\right)^{\top}=\left[\frac{\partial f(x)}{\partial x_{1}}, \ldots, \frac{\partial f(x)}{\partial x_{n}}\right]^{\top}
\end{aligned}
$$

for some $i, j \in\{1, \ldots, n\}$. Let $S \subseteq \mathbb{R}^{n}$, then a property is satisfied for almost all $x \in S$ if it is satisfied for all $x \in S$ except for a set of Lebesgue-measure zero. Almost convergence means convergence for all initial conditions except a set of Lebesgue-measure zero.

\section{B. Practical Stability}

In this paper, we deal with the class of systems that depend on a parameter which we denote by $\omega$. Asymptotic convergence and Lyapunov stability are well known and established notions of stability theory. In this section, we present some standard extensions of these notions. Let $x(t):=x\left(t ; t_{0}, x_{0}, \omega\right)$ denote the solution of the differential equation

$$
\dot{x}=f_{\omega}(t, x)
$$

through $x\left(t_{0}\right)=x_{0}$, where the vector field $f_{\omega}: \mathbb{R} \times \mathbb{R}^{n} \rightarrow$ $\mathbb{R}^{n}$ depends on the parameter $\omega \in(0, \infty)$. In extremum seeking where periodic inputs are steadily applied to the system, it is not possible to establish stability in the sense of Lyapunov. For this purpose, we introduce practical stability which is taken from [3] and [11].

Definition 1: A point $x_{s} \in \mathbb{R}^{n}$ is said to be practically uniformly stable for (1) if for every $\epsilon \in(0, \infty)$ there exists a $\delta \in(0, \infty)$ and $\omega_{0} \in(0, \infty)$ such that for all $t_{0} \in \mathbb{R}$ and for all $\omega \in\left(\omega_{0}, \infty\right)$

$$
x\left(t_{0}\right) \in B\left(x_{s}, \delta\right) \Rightarrow x(t) \in B\left(x_{s}, \epsilon\right), t \in\left[t_{0}, \infty\right) .
$$

Similarly, we define the notion of practical convergence to point.

Definition 2: A point $x_{s} \in \mathbb{R}^{n}$ is said to be practically uniformly attractive for (1) if there exists a $\delta \in(0, \infty)$ such that for every $\epsilon \in(0, \infty)$ there exists a $t_{f} \in[0, \infty)$ and $\omega_{0}$ such that for all $t_{0} \in \mathbb{R}$ and all $\omega \in\left(\omega_{0}, \infty\right)$

$$
x\left(t_{0}\right) \in B\left(x_{s}, \delta\right) \Rightarrow x(t) \in B\left(x_{s}, \epsilon\right), t \in\left[t_{0}+t_{f}, \infty\right) .
$$

Thus, we can define practical local uniform asymptotic stability which is the combination of practical uniform stability and practical uniform attactiveness.

Definition 3: A point $x_{s} \in \mathbb{R}^{n}$ is said to be locally practically uniformly asymptotically stable for (1) if it is practically uniformly stable and practically uniformly attractive.

Since it is impossible to state global convergence to a certain steady state for dynamical systems with multiple steady states we introduce a different notion of convergence which covers the case of convergence to a point for initial conditions in a given set $I$. This set will contain only points which belong to the region of attraction of a certain steady state.

Definition 4: Let $I \subseteq \mathbb{R}^{n}$. A point $x_{s} \in I$ is said to be practically uniformly attractive with respect to $I$ for (1) if for every $\delta, \epsilon \in(0, \infty)$ there exists a $t_{f} \in[0, \infty)$ and $\omega_{0}$ such that for all $t_{0} \in \mathbb{R}$ and all $\omega \in\left(\omega_{0}, \infty\right)$

$$
x\left(t_{0}\right) \in B\left(x_{s}, \delta\right) \cap I \Rightarrow x(t) \in B\left(x_{s}, \epsilon\right), t \in\left[t_{0}+t_{f}, \infty\right) .
$$

The following definitions consider systems which do not depend on a parameter.

Definition 5: Let $I \subseteq \mathbb{R}^{n}$. A point $x_{s} \in I$ is said to be uniformly attractive with respect to $I$ for $\dot{x}=f(t, x)$ if for every $\delta, \epsilon \in(0, \infty)$ there exists a $t_{f} \in[0, \infty)$ such that for all $t_{0} \in \mathbb{R}$

$$
x\left(t_{0}\right) \in B\left(x_{s}, \delta\right) \cap I \Rightarrow x(t) \in B\left(x_{s}, \epsilon\right), t \in\left[t_{0}+t_{f}, \infty\right) .
$$

Furthermore, we define invariance of a set as follows

Definition 6: Let $I \subseteq \mathbb{R}^{n}$. The solutions of $\dot{x}=f(t, x)$ are said to be uniformly invariant with respect to $I$ if for all $t_{0} \in \mathbb{R}$

$$
x\left(t_{0}\right) \in I \Rightarrow x(t) \in I, t \in\left[t_{0}, \infty\right) .
$$

\section{Sphere Worlds}

In the following, we introduce the notion of a sphere world in $\mathbb{R}^{2}$. The term is used in [5] and denotes a workspace which is bounded by a sphere together with spherical obstacles in its interior.

We define the workspace as

$$
W=\left\{x \in \mathbb{R}^{2}: x^{\top} x-r_{0}^{2} \leq 0\right\} .
$$

The obstacles are also bounded by a sphere and are given by

$$
\begin{gathered}
O_{i}=\left\{x \in \mathbb{R}^{2}:\left(x-x_{i}\right)^{\top}\left(x-x_{i}\right)-r_{i}^{2}<0\right\}, \\
i=1, \ldots, m .
\end{gathered}
$$


The free space $S$ is thus defined by

$$
S=W \backslash\left\{\bigcup_{i=1}^{m} O_{i}\right\} .
$$

In terms of functions $\beta_{i}, i=0, \ldots, m$, we define

$$
S=\left\{x \in \mathbb{R}^{2}: \beta_{i}(x) \geq 0, i=0, \ldots, m\right\}
$$

with $\beta_{0}(x)=r_{0}^{2}-x^{\top} x$ and $\beta_{i}(x)=\left(x-x_{i}\right)^{\top}\left(x-x_{i}\right)-r_{i}^{2}$, $i=1, \ldots, m$. We assume that all obstacles are contained in the interior of the workspace, i.e. $r_{0}>0,\left|x_{i}\right|+r_{i}<r_{0}$ and that none of the obstacles intersect $\left|x_{i}-x_{j}\right|>r_{i}+r_{j}, i, j=$ $1, \ldots, m, i \neq j$. Note that the boundary $\partial S$ is the set $\partial S=$ $\left\{x \in S:\right.$ there exists an $i \in\{0, \ldots, m\}$ such that $\beta_{i}(x)=$ $0\}$. Next define the functions

$$
\varphi(x)=\frac{f_{d}(x)}{\left(f_{d}^{k}(x)+\beta(x)\right)^{\frac{1}{k}}}
$$

with $k \in \mathbb{N}$,

$$
f_{d}(x)=\left(x-x_{d}\right)^{\top}\left(x-x_{d}\right)
$$

where $x_{d} \in \operatorname{int}(S)$ denotes the destination point and

$$
\beta(x)=\prod_{i=0}^{m} \beta_{i}(x) .
$$

An illustration of a sphere world is shown in Fig. 1.

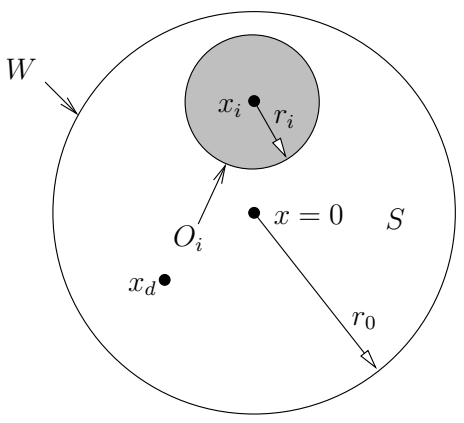

Fig. 1: Sphere World

Note that $\varphi(x) \in[0,1]$ for all $x \in S$ and furthermore $\varphi^{-1}(0)=x_{d}$. We define the sublevel sets of $\varphi$ in $S$ up to $h \in[0,1]$ as follows

$$
S_{h}=\{x \in S: \varphi(x) \leq h\} .
$$

Note that $S_{h}$ is compact and $x_{d} \in S_{h}$ and $S_{h} \subseteq \operatorname{int}(S)$ for all $h \in[0,1)$. Furthermore, we have that $S=S_{1}$.

Definition 7: Let $S \subseteq \mathbb{R}^{2}$ be a compact, connected, analytic manifold with boundary. A function $\varphi: S \rightarrow[0,1]$ is a navigation function if it is

1) Analytic on $S$,

2) Polar on $S$, i.e. $x_{d}$ is the unique minimizer $\operatorname{in} \operatorname{int}(S)$,

3) Morse on $S$,

4) Admissible on $S$, i.e. $\varphi(x)=1$ for $x \in \partial S$.

We refer to Definition 1 in [5] for more information. As already mentioned in the introduction, it turns out that these properties are crucial for the methodology in the following sections. The set of critical points of $\varphi$ is defined as

$$
X_{c}=\{x \in S: \nabla \varphi(x)=0\} .
$$

By Theorem 4 in [5] $\varphi$ in (7) is a valid navigation function for finitely many obstacles, for $x_{d} \in \operatorname{int}(S)$ and a sufficiently large integer $k \in \mathbb{N}$. A constructive procedure for determining $k$ is given in [5].

Remark 1: Since $\varphi$ is a Morse function on $S$ and $S$ is compact, the set $X_{c}$ consists of finitely many isolated points (see Corollary 2.19 in [9]). Moreover, by Proposition 3.3 in [5] all critical points are in $\operatorname{int}(S)$.

Note that due to the Remark p. 424 in [5] $\varphi$ is actually analytic in an open set containing $S$.

\section{MAIN RESULT}

In the following, we consider the extremum seeking system and the corresponding Lie bracket system. First, consider the extremum seeking system in Fig. 2 (see e.g. [3]), where $\varphi$ is defined above. We use $x=\left[x_{1}, x_{2}\right]^{\top} \in \mathbb{R}^{2}$ and $z=\left[z_{1}, z_{2}\right]^{\top} \in \mathbb{R}^{2}$.

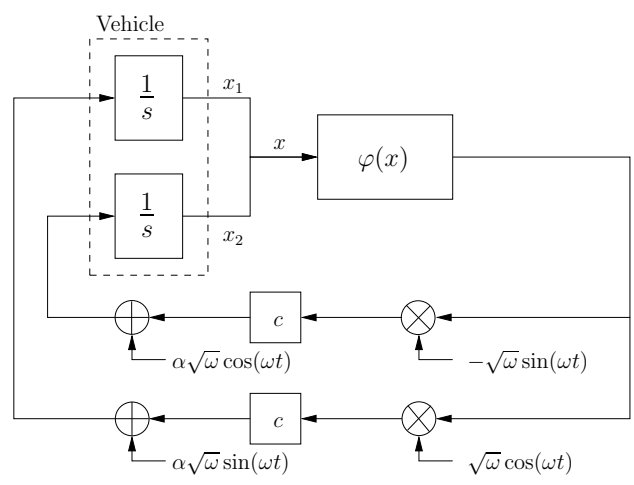

Fig. 2: Extremum Seeking Vehicle

The equations of motion are given by

$$
\left[\begin{array}{c}
\dot{x}_{1} \\
\dot{x}_{2}
\end{array}\right]=\underbrace{\left[\begin{array}{c}
\alpha \\
-c \varphi(x)
\end{array}\right]}_{b_{1}(x)} \sqrt{\omega} \underbrace{\sin (\omega t)}_{u_{1}(\omega t)}+\underbrace{\left[\begin{array}{c}
c \varphi(x) \\
\alpha
\end{array}\right]}_{b_{2}(x)} \sqrt{\omega} \underbrace{\cos (\omega t)}_{u_{2}(\omega t)}
$$

where we assume for the rest of the paper that $\alpha, c \in(0, \infty)$ and $\varphi$ is a navigation function. Second, this system can be interpreted as an input-affine system with inputs $u_{1}$ and $u_{2}$. Following the ideas of [3] we calculate the corresponding Lie bracket system of (12), i.e. $\dot{z}=-\frac{1}{2}\left[b_{1}, b_{2}\right](z)=$ $-\frac{1}{2}\left(\frac{\partial b_{2}(x)}{\partial x} b_{1}(x)-\frac{\partial b_{1}(x)}{\partial x} b_{2}(x)\right)$, where $\frac{\partial b_{i}(x)}{\partial x}$ denotes the Jacobian of $b_{i}, i=1,2$. This yields the so-called Lie bracket system

$$
\begin{aligned}
{\left[\begin{array}{c}
\dot{z}_{1} \\
\dot{z}_{2}
\end{array}\right] } & =-\frac{1}{2}\left[\begin{array}{c}
\alpha c \nabla_{1} \varphi(z)-c^{2} \varphi(z) \nabla_{2} \varphi(z) \\
\alpha c \nabla_{2} \varphi(z)+c^{2} \varphi(z) \nabla_{1} \varphi(z)
\end{array}\right] \\
& =-\underbrace{\frac{1}{2}\left[\begin{array}{cr}
\alpha c & -c^{2} \varphi(z) \\
c^{2} \varphi(z) & \alpha c
\end{array}\right]}_{G}\left[\begin{array}{l}
\nabla_{1} \varphi(z) \\
\nabla_{2} \varphi(z)
\end{array}\right]
\end{aligned}
$$


where $G$ is positive definite (but not symmetric), since $z^{\top} G z=\frac{1}{2} \alpha c\left(z_{1}^{2}+z_{2}^{2}\right)>0$ for all $z=\left[z_{1}, z_{2}\right]^{\top} \in \mathbb{R}^{2} \backslash\{0\}$.

The main idea of the paper is to relate the qualitative behavior of (12) to the qualitative behavior of (13). In Subsection III-A we show that (13) converges for almost all initial conditions to the destination point $x_{d}$ while avoiding the obstacles. This claim follows by showing that the set $\operatorname{int}(S)$ is invariant. In Subsection III-B we guarantee obstacle avoidance and almost uniform attractivity of the destination point $x_{d}$ for the extremum seeking system (12) using results from [3].

\section{A. Invariance and Stability of the Lie Bracket System}

In the first step we establish properties of the Lie bracket system (13) which will be important for the analysis of the extremum seeking system (12). We show that the interior of $S$ is uniformly invariant for (13) and that for almost all initial conditions, the solutions of (13) converge to the destination point $x_{d}$. The set of steady states can easily be calculated.

Lemma 1: The set of steady states of (13) is exactly $X_{c}$ in (11).

In order to guarantee obstacle avoidance for (13), we must show that the interior of the free space $S$ is uniformly invariant.

Lemma 2: The solutions of (13) are uniformly invariant with respect to $S_{h}$ for every $h \in[0,1)$. Furthermore, the solutions of (13) are uniformly invariant with respect to $\operatorname{int}(S)$ and every solution of (13) initialized in $\operatorname{int}(S)$ converges to the set $X_{c}$.

Proof: First, we show by using a Lyapunov argument, that for every $h \in[0,1)$ the set $S_{h}$ is invariant for (13). Since for every $h \in[0,1)$ the set $S_{h}$ is compact, we can use the Lyapunov function candidate $V=\varphi$ which has compact level sets in $S_{h}$ and calculate its derivative along the solutions of (13). We obtain with $\alpha, c \in(0, \infty)$

$$
\begin{aligned}
\dot{V}= & \frac{1}{2} \nabla_{1} \varphi(z)\left(-\alpha c \nabla_{1} \varphi(z)+c^{2} \varphi(z) \nabla_{2} \varphi(z)\right) \\
& +\frac{1}{2} \nabla_{2} \varphi(z)\left(-\alpha c \nabla_{2} \varphi(z)-c^{2} \varphi(z) \nabla_{1} \varphi(z)\right) \\
= & -\frac{\alpha c}{2}\left(\left(\nabla_{1} \varphi(z)\right)^{2}+\left(\nabla_{2} \varphi(z)\right)^{2}\right) \leq 0
\end{aligned}
$$

Second, since $\dot{V}=\dot{\varphi}$ is negative semi-definite and thus $\varphi$ is non-increasing along the solutions of (13), we conclude that the set $S_{h}$ is invariant for every $h \in[0,1)$. Third we show convergence to the set $X_{c}$. Note that for every $z\left(t_{0}\right) \in \operatorname{int}(S)$ there exists a $\tilde{h} \in[0,1)$ such that the solution $z(t)$ initialized at $z\left(t_{0}\right)$ is invariant with respect to $S_{\tilde{h}} \subseteq \operatorname{int}(S)$. Again, since $S_{\tilde{h}}$ is compact we conclude with LaSalle's Invariance Principle that the solutions of (13) converge to the largest invariant set where $\dot{V} \equiv 0$ which is $X_{c}$.

Since $\varphi$ is Morse, all critical points in $X_{c}$ are non-degenerate. Thus, since the system matrix of the linearization of $\dot{y}=$ $-\nabla \varphi(y)$ around every point $x \in X_{c}$ is given by the negative Hessian of $\varphi$, all points in $x \in X_{c}$ are hyperbolic steady states of $\dot{y}=-\nabla \varphi(y)$. In the proof of the following lemma, we show that this is also true for the steady states of (13).
Lemma 3: Consider (13). Every point in the set $X_{c}$ in (11) is a hyperbolic steady state and all points are unstable except $x_{d}$ which is locally asymptotically stable.

Proof: Note that the steady states of $\dot{y}=-\nabla \varphi(y)$ in $S$ is exactly $X_{c}$. We show that the local stability property of every point in $X_{c}$ is the same for $\dot{y}=-\nabla \varphi(y)$ and (13).

First, we compare the coefficients of the respective characteristic polynomials of the system matrices of the linearizations of $\dot{y}=-\nabla \varphi(y)$ and (13). The system matrix of the linearization of $\dot{y}=-\nabla \varphi(y)$ coincides with the negative Hessian of $\varphi$ evaluated at some $x \in X_{c}$ and which is given by

$$
H(x)=-\left[\begin{array}{cc}
\nabla_{1}^{2} \varphi(x) & \nabla_{1,2} \varphi(x) \\
\nabla_{1,2} \varphi(x) & \nabla_{2}^{2} \varphi(x)
\end{array}\right] .
$$

Since $S \subseteq \mathbb{R}^{2}$ the eigenvalues of $H(x)$ are exactly determined by $\operatorname{det}(H(x))=\nabla_{1}^{2} \varphi(x) \nabla_{2}^{2} \varphi(x)-\left(\nabla_{1,2} \varphi(x)\right)^{2}$ and $\operatorname{trace}(H(x))=-\left(\nabla_{1}^{2} \varphi(x)+\nabla_{2}^{2} \varphi(x)\right)$.

Next, the system matrix of the linearization of (13) evaluated at some $x \in X_{c}$ is given by

$$
A(x)=-\frac{\alpha c}{2}\left[\begin{array}{cc}
\nabla_{1}^{2} \varphi-\frac{c}{\alpha} \varphi \nabla_{1,2} \varphi & \nabla_{1,2} \varphi-\frac{c}{\alpha} \varphi \nabla_{2}^{2} \varphi \\
\nabla_{1,2} \varphi+\frac{c}{\alpha} \varphi \nabla_{1}^{2} \varphi & \nabla_{2}^{2} \varphi+\frac{c}{\alpha} \varphi \nabla_{1,2} \varphi
\end{array}\right],
$$

where we omitted the arguments of $\varphi=\varphi(x)$. One can verify that $\operatorname{det}(A(x))=\frac{1}{4}\left(\alpha^{2} c^{2}+c^{4} \varphi(x)^{2}\right)\left(\nabla_{1}^{2} \varphi(x) \nabla_{2}^{2} \varphi(x)-\right.$ $\left.\left(\nabla_{1,2} \varphi(x)\right)^{2}\right)=\frac{1}{4}\left(\alpha^{2} c^{2}+c^{4} \varphi(x)^{2}\right) \operatorname{det}(H(x))$ and $\operatorname{trace}(A(x))=-\frac{\alpha c}{2}\left(\nabla_{1}^{2} \varphi(x)+\nabla_{2}^{2} \varphi(x)\right)=\frac{\alpha c}{2} \operatorname{trace}(H(x))$. The characteristic polynomial of $A(x)$ is given by

$$
s^{2}+\underbrace{\frac{\alpha c}{2} \operatorname{trace}(H(x))}_{\operatorname{trace}(A(x))} s+\underbrace{\frac{\alpha^{2} c^{2}+c^{4} \varphi(x)^{2}}{4} \operatorname{det}(H(x))}_{\operatorname{det}(A(x))} \text {. }
$$

We compare this with the characteristic polynomial of $H(x)$

$$
s^{2}+\operatorname{trace}(H(x)) s+\operatorname{det}(H(x)) .
$$

Since $\alpha, c \in(0, \infty)$ and $\varphi(x) \in[0,1]$ for all $x \in X_{c} \subseteq S$, the coefficients of the characteristic polynomials of $A(x)$ have the same sign as the coefficients of the characteristic polynomial of $H(x)$.

Second, we will exclude degenerate steady states. Note that $\frac{\alpha c}{2}$ and $\frac{\left(\alpha^{2} c^{2}+c^{4} \varphi(x)^{2}\right)}{4}$ in (17) are strictly positive. Since (17) can have only real or complex conjugated roots, there are only two possibilities that (17) has at least one root on the imaginary axis:

$$
\begin{aligned}
& \text { 1) } \frac{\alpha c}{2} \operatorname{trace}(H(x))=0 \text { and } \frac{\alpha^{2} c^{2}+c^{4} \varphi(x)^{2}}{4} \operatorname{det}(H(x)) \geq 0 \\
& \text { 2) } \frac{\alpha^{2} c^{2}+c^{4} \varphi(x)^{2}}{4} \operatorname{det}(H(x))=0 .
\end{aligned}
$$

Both cases can be excluded, since $\varphi$ is Morse and thus the roots of (18) are hyperbolic.

Third, by the same argument, it follows that (17) has roots in the open left half-plane if and only if (18) has roots in the open left half-plane. Moreover, by Proposition 3.9 in [5], (18) has roots in the open left half plane only if $x=x_{d}$.

Theorem 1: Consider (13). There exists a set $S^{0}$ of Lebesgue-measure zero such that the destination point $x_{d}$ is locally asymptotically stable and uniformly attractive with respect to $\operatorname{int}(S) \backslash S^{0}$ and uniformly invariant with respect to $\operatorname{int}(S)$. 
Proof: With Lemma 2 we conclude that for all initial conditions the solutions of (13) converge to the set $X_{c}$.

Since $x_{d}$ is the unique asymptotically stable steady state for (13) it is left to show that the region of attraction of all other critical points is of measure zero. By Lemma 3 all points in $X_{c}$ are hyperbolic steady states of (13) and all steady states except the destination point $x_{d}$ are unstable.

Since $\varphi$ is analytic, it is smooth and with Theorem 0.8 in [12] the flow of (13) is smooth. Using the Global Stable Manifold Theorem e.g. Theorem 6.2 in [12] we conclude that the dimension $\operatorname{dim}\left(W^{s}(x)\right)$ of the global stable manifold $W^{s}(x)$ of every unstable steady state $x \in X_{c} \backslash\left\{x_{d}\right\}$ is $\operatorname{dim}\left(W^{s}(x)\right)=1$ since its tangent space $T_{x} W^{s}(x)$ is of the same dimension as the stable subspace of the linearization at $x$. Thus, every global stable manifold is of measure zero. Since there are finitely many points in $X_{c}$, the union of all stable manifolds is $S^{0}=\bigcup_{x \in X_{c} \backslash\left\{x_{d}\right\}} W^{s}(x)$ of all unstable steady states is of measure zero and thus the region of attraction of the stable steady state $x_{d}$ is $\operatorname{int}(S) \backslash S^{0}$. Uniform invariance with respect to the set $\operatorname{int}(S)$ follows by Lemma 2 .

\section{B. Invariance and Stability of the Extremum Seeking System}

In the second step, we relate the properties of the solutions of (13) to the solutions of (12) by using the trajectory approximation for extremum seeking introduced in [3]. By Theorem 1 in [3] it is possible to approximate arbitrarily close the trajectories of (13) by (12) in the $L_{\infty}$-sense for an arbitrary large but finite time-interval if the value of the parameter $\omega$ is sufficiently large. This can be exploited in order to assure obstacle avoidance for (12). Thus, if $\omega$ is chosen such that the distance between the trajectories of (12) and (13) is less than the distance of the trajectory of (13) to the boundary $\partial S$, one can conclude that the solutions of (12) stay in $\operatorname{int}(S)$ for a fixed time. Since in this setup a special case of Theorem 1 in [3] is needed, we state it as a lemma.

Lemma 4: For every $h \in[0,1)$, every $D \in(0, \infty)$ and every $t_{f} \in(0, \infty)$, there exists an $\omega_{0} \in(0, \infty)$ such that for every $\omega \in\left(\omega_{0}, \infty\right)$, for every $t_{0} \in \mathbb{R}$ and every $x_{0} \in S_{h}$ in (10) there exist unique solutions $x(t)$ of (12) and $z(t)$ of (13) through $x\left(t_{0}\right)=z\left(t_{0}\right)=x_{0}$ which satisfy

$$
|x(t)-z(t)|<D \text { and } x(t) \in \operatorname{int}(S), t \in\left[t_{0}, t_{0}+t_{f}\right] .
$$

Because of space restrictions we omit the proof of this lemma. However, it goes along the same lines as Theorem 1 in [3].

Remark 2: Note that by Lemma 2 the solutions of (13) are invariant with respect to the set $S_{h}$ and since $\varphi^{-1}(1)=\partial S$ and $S_{h} \subseteq \operatorname{int}(S)$ for any $h \in[0,1)$ we have that the distance $e=\inf _{r \in S_{h}, s \in \partial S}|r-s|$ from $S_{h}$ to the boundary $\partial S$ exists and is strictly positive. Thus, by making $D$ sufficiently small and $\omega$ sufficiently large the solutions of (12) exist and stay in $\operatorname{int}(S)$ for $t \in\left[t_{0}, t_{0}+t_{f}\right]$.

Theorem 2: Consider (12). The destination point $x_{d}$ is locally practically uniformly asymptotically stable and there exists a set $S^{0}$ of Lebesgue-measure zero such that for every $h \in[0,1)$ the destination point $x_{d}$ is practically uniformly attractive with respect to $S_{h} \backslash S^{0}$. Furthermore, for every $h \in[0,1)$ there exists an $\omega_{0} \in(0, \infty)$ such that for all $t_{0} \in \mathbb{R}$ and all $\omega \in\left(\omega_{0}, \infty\right)$

$$
x\left(t_{0}\right) \in S_{h} \backslash S^{0} \Rightarrow x(t) \in \operatorname{int}(S), t \in\left[t_{0}, \infty\right),
$$

where $x(t)$ denotes the solution of (12).

Proof: We prove the first statement. Since by Lemma 3 the destination point is locally asymptotically stable for (13) and the vector field is time-invariant we have that it is locally uniformly asymptotically stable for (13). We conclude with Theorem 2 in [3] that the destination point is locally practically uniformly asymptotically stable. Strictly speaking, in order to apply Theorem 2 in [3], one has to show that this theorem holds also if Assumptions A1 - A2 in [3] are satisfied only on the domain $S_{h}$ and not on the whole domain $\mathbb{R}^{2}$.

Next, we prove the second statement. In the following we denote the solutions of (12) by $x(t)$ and the solutions of (13) by $z(t)$. Let $\delta, \epsilon \in(0, \infty)$ and $h \in[0,1)$ be given. By the local practical uniform stability and since $x_{d} \in \operatorname{int}(S)$, there exist an $\omega_{1} \in(0, \infty)$ and a $\delta_{1} \in(0, \infty)$ such that for all $t_{0} \in \mathbb{R}$ and all $\omega \in\left(\omega_{1}, \infty\right)$

$$
\begin{aligned}
x\left(t_{0}\right) & \in B\left(x_{d}, \delta_{1}\right) \\
& \Rightarrow x(t) \in B\left(x_{d}, \epsilon\right) \cap \operatorname{int}(S), t \in\left[t_{0}, \infty\right) .
\end{aligned}
$$

Choose $\epsilon_{1} \in\left(0, \delta_{1}\right)$. By the uniform invariance of $S_{h}$ for (13) in Lemma 2 and the uniform attractivity of $x_{d}$ with respect to $\operatorname{int}(S) \backslash S^{0}$ for (13) in Theorem 1 there exist a $S^{0} \in \mathbb{R}^{2}$ of measure zero and a $t_{f} \in(0, \infty)$ such that for all $t_{0} \in \mathbb{R}$ we have that

$$
\begin{aligned}
z\left(t_{0}\right) & \in B\left(x_{d}, \delta\right) \cap S_{h} \backslash S^{0} \\
& \Rightarrow z(t) \in B\left(x_{d}, \epsilon_{1}\right) \cap S_{h}, t \in\left[t_{0}+t_{f}, \infty\right) .
\end{aligned}
$$

Using Lemma 4 we choose $D=\delta_{1}-\epsilon_{1}$ and $h, t_{f}$ as above which yields an $\omega_{2} \in(0, \infty)$ such that for all $x\left(t_{0}\right)=$ $z\left(t_{0}\right) \in S_{h}$ we have $|x(t)-z(t)|<D$ and $x(t) \in \operatorname{int}(S)$, $t \in\left[t_{0}, t_{0}+t_{f}\right]$. Together with (22) we have for all $x\left(t_{0}\right)=$ $z\left(t_{0}\right) \in B\left(x_{d}, \delta\right) \cap S_{h} \backslash S^{0}$ that $x\left(t_{0}+t_{f}\right) \in B\left(x_{d}, \delta_{1}\right)$. By the local practical uniform stability proven above, we have that $x(t) \in B\left(x_{d}, \epsilon\right) \cap \operatorname{int}(S)$ for $t \in\left[t_{0}+t_{f}, \infty\right)$. We conclude that for every $\delta, \epsilon \in(0, \infty)$ there exists an $\omega_{3}=\max \left\{\omega_{1}, \omega_{2}\right\}$ such that for all $t_{0} \in \mathbb{R}$ and all $\omega \in\left(\omega_{3}, \infty\right)$

$$
\begin{aligned}
x\left(t_{0}\right) & \in B\left(x_{d}, \delta\right) \cap S_{h} \backslash S^{0} \\
& \Rightarrow x(t) \in B\left(x_{d}, \epsilon\right) \cap \operatorname{int}(S), t \in\left[t_{0}+t_{f}, \infty\right) .
\end{aligned}
$$

Furthermore, since we can choose $\delta \in(0, \infty)$ such that $S_{h} \backslash S^{0} \subseteq B\left(x_{d}, \delta\right)$ we have that $x(t) \in \operatorname{int}(S), t \in$ $\left[t_{0}, t_{0}+t_{f}\right]$ and $x(t) \in B\left(x_{d}, \epsilon\right) \cap \operatorname{int}(S), t \in\left[t_{0}+t_{f}, \infty\right)$. Thus $x(t) \in \operatorname{int}(S), t \in\left[t_{0}, \infty\right)$. This was the last statement to prove.

\section{Discussion}

In the following, we give some remarks on the results above. 
Remark 3: We assume that the value of $\varphi$ is available at each point in the free space. Although $\varphi$ is an artificial function and not directly measurable through sensors, its value can be calculated using algebraic operations involving the distances to the obstacles and to the destination point $x_{d}$.

Remark 4: The extremum seeking scheme in Fig. 2 does not contain a washout-filter as it is usually the case (see e.g. [3]). The filter was removed since $\varphi$ is constructed in such a way that $\varphi\left(x_{d}\right)=0$ and thus there is no constant offset at this point and no need for a filter.

Remark 5: So far there is no procedure for estimating a good choice for $\omega_{0}$ in Theorem 2 which guarantees convergence. Since $\omega_{0}$ depends on the choice of $d, \epsilon$ and $k$ it is not trivial to give a constructive procedure for the values of these parameters.

Remark 6: It is shown in Theorem 2 that $x_{d}$ is practically uniformly attractive with respect to $\operatorname{int}(S) \backslash S^{0}$ for (12) where $S^{0}$ is of measure zero. Due to the periodic excitations in the extremum seeking system, one can expect that for all initial conditions in $\operatorname{int}(S)$ the extremum seeking system (12) converges to the destination point. We refer to the next section for a numerical example.

Remark 7: The methodology used for the analysis of (13) herein differs from [7], [2]. Therein, almost practical convergence was shown using Rantzer's Dual Lyapunov Theorem [13]. However, as pointed out in [1], [15], for non-degenerate critical points an alternative approach is to linearize the vector field and conclude almost convergence using the Stable Manifold Theorem. A similar approach was already used in [4] and [5].

\section{EXAMPLES}

In this section we show two scenarios which are meant to highlight some facts of the previous results.

In the first example we consider a sphere world with three obstacles. The obstacles are located at $x_{1}=[0.5,0.5]^{\top}$, $x_{2}=[-0.8,0.5]^{\top}$ and $x_{3}=[0.5,-0.5]^{\top}$. We choose the parameters $r_{0}=2, r_{1}=r_{2}, r_{3}=0.4, k=4$. The destination point is at the origin, i.e. $x_{d}=[0,0]^{\top}$. The parameters of the extremum seeking are chosen $\alpha=c=1$. In Fig. 3a the resulting trajectory for the extremum seeking sytem (12) with $\omega=100$ and the Lie bracket system (13) as well as for the gradient system $\dot{x}=-\nabla \varphi(x)$ are depicted. We can see that the Lie bracket system as well as the gradient system and the extremum seeking converge to the destination point and avoid the obstacles. This is in the sense of Theorem 2.

In the next example we consider only one obstacle at $x_{1}=[1,0]^{\top}$ and with parameters $r_{0}=3, r_{1}=0.4, k=4$. The destination point is again at the origin. For the extremum seeking we choose $\alpha=0.9, c=0.5$ and $\omega=50$. We choose an initial condition which is at an unstable saddle point of $\varphi$. We see in Fig. 3b that this is a steady state for the Lie bracket system as well as for the gradient system. However, the extremum seeking system converges to the destination point. An explanation for this behavior is given in Remark 6 and shows that in practice one can expect the system converges for all initial conditions to the destination point.

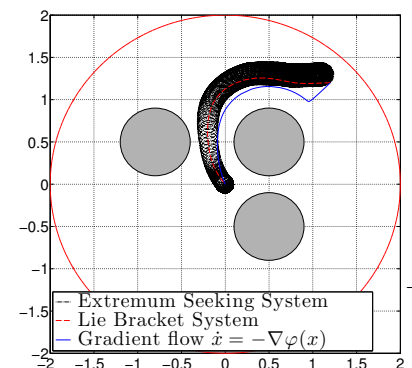

(a) $\omega=100$

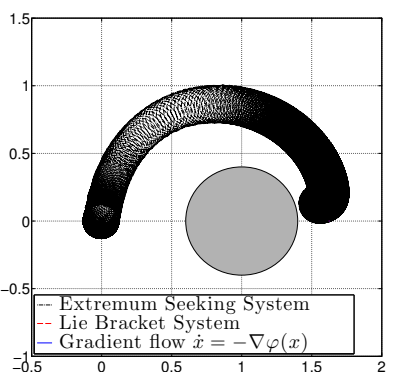

(b) $\omega=50$
Fig. 3: Simulation examples

\section{Summary AND OUTLOOK}

The main result was to establish guaranteed practical convergence and obstacle avoidance for a single-integrator extremum seeking vehicle in a sphere world. Our approach builds up on the navigation function approach and exploits the idea of Lie bracket approximation for extremum seeking vehicles.

In this paper, the setup was restricted to two dimensions and a single agent. Future research considers the generalization of the approach towards higher dimensions and multiple agents. Furthermore, it is of high interest to derive practical conditions for the choice of parameter $\omega$.

\section{ACKNOWLEDGEMENTS}

We thank Gerd Simon Schmidt for fruitful discussions.

\section{REFERENCES}

[1] D. Angeli. An almost global notion of input-to-state stability. IEEE Transactions on Automatic Control, 49(6):866 - 874, 2004.

[2] D. V. Dimarogonas, S. G. Loizou, K. J. Kyriakopoulos, and M. M. Zavlanos. A feedback stabilization and collision avoidance scheme for multiple independent non-point agents. Automatica, 42(2):229 243, 2006.

[3] H. B. Dürr, M. S. Stanković, C. Ebenbauer, and K. H. Johansson. Lie Bracket Approximation of Extremum Seeking Systems. To appear in Automatica, http://dx.doi.org/10.1016/j.automatica.2013.02.016.

[4] U. Helmke and J. B. Moore. Optimization and Dynamical Systems. Springer, 1996.

[5] D. Koditschek and E. Rimon. Robot navigation functions on manifolds with boundary. Advances in Applied Mathematics, 11:412 - 442, 1990.

[6] M. Krstić and K. B. Ariyur. Real-Time Optimization by ExtremumSeeking Control. Wiley-Interscience, 2003.

[7] S. Loizou and A. Jadbabaie. Density functions for navigation-functionbased systems. IEEE Transactions on Automatic Control, 53(2):612 617, 2008.

[8] S. Loizou and K. Kyriakopoulos. Navigation of multiple kinematically constrained robots. Robotics, IEEE Transactions on, 24(1):221 - 231, 2008.

[9] Y. Matsumoto. An Introduction to Morse Theory, volume 208. American Mathematical Society, 2002.

[10] J. Milnor. Morse Theory. Princeton University Press, 1963.

[11] L. Moreau and D. Aeyels. Practical stability and stabilization. IEEE Transactions on Automatic Control, 45(8):1554 - 1558, 2000.

[12] J. Palis and W. de Melo. Geometric Theory of Dynamical Systems. Springer, 1982.

[13] A. Rantzer. A dual to Lyapunov's stability theorem. Systems and Control Letters, 42:161 - 168, 2001.

[14] Y. Tan, D. Nešić, and I. Mareels. On non-local stability properties of extremum seeking control. Automatica, 42:889 - 903, 2006.

[15] J. F. Vasconcelos, A. Rantzer, C. Silvestre, and P. Oliveira. Combination of Lyapunov and Density Functions for Stability of Rotational Motion. IEEE Transactions on Automatic Control, 2011.

[16] C. Zhang and R. Ordóñez. Extremum-Seeking Control and Applications: A Numerical Optimization-Based Approach. Springer, 2012. 